\title{
Hormone replacement therapy may increase the risk of early miscarriage during frozen embryo transfer cycles in patients with a history of cesarean section: a retrospective cohort study
}

\author{
Run-xin Gan ${ }^{1}$, Yuan $\mathrm{Li}^{1}$, Juan Song ${ }^{1}$, Quan Wen ${ }^{1}$, Guang-xiu Lu ${ }^{1}$, Ge Lin ${ }^{1}$, and Fei Gong ${ }^{1}$ \\ ${ }^{1}$ Affiliation not available
}

September 24, 2021

\begin{abstract}
Objective: To investigate the efficacies of three cycle regimens in women receiving FET with a history of CS: natural cycle (NC) treatment, hormone replacement therapy (HRT) and treatment with gonadotropin-releasing hormone agonist (GnRH-a) + HRT). Design: Retrospective cohort study. Setting: University-affiliated center. Population: Patients $(\mathrm{N}=6,159)$ with a history of CS who fulfilled the inclusion criteria were enrolled in the study from January 2014 to December 2019. Methods: Reproductive outcomes of patients in the NC $(\mathrm{n}=4,306)$ versus HRT $(\mathrm{n}=1,007)$ versus GnRH-a + HRT groups $(\mathrm{n}=846)$ were compared. Main Outcome Measure: The main outcome measure was the live birth rate per embryo transfer (ET). Results: The unadjusted odds of the miscarriage rate of singleton pregnancies were also significantly higher in the HRT-group compared with the NC-group (25.5\% versus $20.4 \%$, respectively). After adjusting for possible confounding factors, the early miscarriage rate and the miscarriage rate of singleton pregnancies remained significantly higher in the HRT-group than the NC-group. The clinical pregnancy rates in the NC-, HRT- and GnRH-a + HRT-groups of women with a history of CS was $48.8 \%, 48 \%$ and $47.1 \%$, respectively, and the live birth rates were $37 \%, 34.1 \%$ and $35.7 \%$, respectively. Conclusion(s): In women undergoing FET with a history of CS, HRT for endometrial preparation was associated with a higher early miscarriage rate, albeit after statistical adjustment for confounding factors. Funding: The National Science Foundation of China (81501328). Key Words: Caesarean section, endometrial preparation, frozen embryo transfer, miscarriage
\end{abstract}

\section{Hosted file}

manuscript(1).pdf available at https://authorea.com/users/435527/articles/538484-hormonereplacement-therapy-may-increase-the-risk-of-early-miscarriage-during-frozen-embryotransfer-cycles-in-patients-with-a-history-of-cesarean-section-a-retrospective-cohortstudy

\section{Hosted file}

Tables.doc available at https://authorea.com/users/435527/articles/538484-hormonereplacement-therapy-may-increase-the-risk-of-early-miscarriage-during-frozen-embryotransfer-cycles-in-patients-with-a-history-of-cesarean-section-a-retrospective-cohortstudy 